\title{
The partial mediation effect of satisfaction with social support and coping effectiveness on health-related quality of life and perceived stress long-term after heart transplantation
}

\author{
This article was published in the following Dove Press journal: \\ Nursing: Research and Reviews \\ 5 December 2014 \\ Number of times this article has been viewed
}

\section{Connie White-Williams' \\ Kathleen L Grady² \\ Pariya Fazeli' \\ Susan Myers' \\ Linda Moneyham ${ }^{3}$ \\ Karen Meneses ${ }^{3}$ \\ Bruce Rybarczyk ${ }^{4}$ \\ 'University of Alabama Medical Center, Birmingham, AL, USA; \\ ${ }^{2}$ Division of Cardiac Surgery, \\ Department of Surgery, Feinberg School of Medicine, Northwestern \\ University, Chicago, IL, USA; ${ }^{3}$ School of Nursing, University of Alabama at Birmingham, Birmingham, $\mathrm{AL}$, USA; ${ }^{4}$ Department of Psychology, Clinical Psychology Program, Virginia Commonwealth University, Richmond, VA, USA}

\begin{abstract}
The purpose of this research was to examine whether satisfaction with social support and coping effectiveness mediate the relationship between stress and health-related quality of life (HRQOL) 5 years after heart transplantation. Data were collected from 212 heart transplant patients ( $80 \%$ male, $92 \%$ white, mean age 59.9 years) at four United States sites using the Heart Transplant Stressor Scale, Social Support Index, Jalowiec Coping Scale, and Quality of Life Index. Using Baron and Kenny's approach, a series of regression equations for mediation revealed that both satisfaction with social support and coping partially mediated the relationship between perceived stress and HRQOL. Understanding the relationships of social support, stress, and coping on patients' HRQOL is important for the development of interventions to provide optimal patient care.
\end{abstract}

Keywords: heart transplantation, social support, coping, stress, mediation

\section{Introduction}

Heart transplantation is a life-saving therapy for patients with advanced heart failure. The complexities of the medical regimen highlight the importance of understanding the interplay between psychosocial outcomes such as health-related quality of life (HRQOL), social support, coping, and perceived stress. Long term after transplant, patients continue to cope with daily regimens that include adhering to medications, following diet and exercise plans, and attending follow-up appointments. ${ }^{1-3}$ In addition, while the immediate complications of rejection and infection decrease, the long-term comorbidities of coronary vasculopathy and malignancies increase. ${ }^{1,2}$ Other long-term issues such as osteroporosis, hypertension, and gout may also impact HRQOL. ${ }^{2}$ These transplant-specific conditions make balancing medical management and psychosocial outcomes a lifelong journey. These later comorbidities that transplant patients deal with may impact the need for additional satisfactory social support, and optimal stress and coping strategies.

\section{Perceived stress}

Perceived stress is defined as the degree to which the situations in one's life are viewed as stressful. Researchers have shown psychological stress among heart transplant patients is not only determined by clinical health status, but that it could also be influenced by personal and environmental variables, self-care behaviors, symptoms, and social support. ${ }^{4-7}$
Correspondence: Connie White-Williams 1056 Jefferson Tower, Center for Nursing Excellence, 625 19th Street South,

Birmingham, AL 35249, USA

Tel + I 205975861 I

Fax +I 2059967410

Email cwwilli@uabmc.edu 
Most reported studies examining stress have focused on symptom distress, stress related to the medical regimen, depression, and anxiety. ${ }^{3,4}$ For instance, several researchers revealed a link between social support, medication adherence, and health. ${ }^{3,5-7}$ Sayers et $\mathrm{al}^{7}$ examined the role of social support and self-care in 74 heart failure patients and found that perceived social support was associated with better medication adherence and other self-care activities. The authors found that emotional support may alleviate stress, thereby having beneficial effects in chronic situations.

\section{Social support and coping}

For this study, social support is defined as the degree of perceived satisfaction with social support provided by others. ${ }^{8,9}$ Tangible support involves the condition of providing material support such as monetary assistance. ${ }^{8,9}$ Emotional support involves the provision of closeness, and the ability to confide feelings to someone. ${ }^{8,9}$ Several studies ${ }^{4,10-13}$ have examined perceived satisfaction with social support and perceived stress in chronic illness populations, showing that perceived satisfaction with social support either has a direct or indirect effect on physical and mental health. Further, the literature on satisfaction with social support has demonstrated that satisfaction either has mediating ${ }^{4,11,12}$ or moderating effects on health outcomes. ${ }^{14-16}$ However, contradicting reports have been found in studies examining social support and cardiovascular disease; yet, no studies were found that explicitly examined the mediation relationship between coping and social support after heart transplantation. ${ }^{10}$

Coping is another factor that has been implicated in the chronic illness literature and has been defined as the process by which the "individual manages the demands of the person-environmental relationship that are appraised as stressful and the emotions they generate". ${ }^{14}$ Studies have shown conflicting results regarding whether coping moderates or mediates the effect of stress on psychosocial outcomes. ${ }^{17-20}$ While several researchers have shown that the ability to cope effectively and social support resources can positively impact stressful life events, ${ }^{9,21-23}$ few studies ${ }^{5,7}$ have actually addressed the relationship between coping and social support in the context of heart transplantation exclusively.

\section{Purpose}

Although there is evidence in various chronic disease populations of the relationships of these psychosocial factors, there is less information in the heart transplant population. Therefore, we aimed to examine whether satisfaction with social support and coping effectiveness would mediate the relationship between stress and HRQOL. In this study, quality of life was defined as the effect of an illness and its consequent therapy upon a patient, as perceived by the patient's satisfaction with aspects of life that are important to them. ${ }^{24,25}$ The works of House (social support), and Lazarus and Folkman's stress and coping theory guided this study. ${ }^{8,14}$

\section{Methods}

This study was an original research question from a secondary analysis of data collected as part of two larger parent studies. The first dataset included psychosocial data from the Heart Transplant Quality of Life Outcomes Study, ${ }^{26}$ which was conducted from July 1, 1999 to June 30, 2004. The second dataset included medical and clinical data compiled from the Cardiac Transplant Research Database, which was an event-driven database that collects information on transplant recipients such as immunosuppression, rejection episodes, infection, evidence of cardiac allograft vasculopathy or malignancy, and death. ${ }^{1}$ The analyses in the current study are cross-sectional and include a cohort of heart transplant recipients 5 years post-transplantation. There were 555 patients enrolled in this study in total, all of which were between 5 years and 10 years post-transplantation. We chose to use the 5-year cohort for this study, as the sample size was the largest across all of the time points.

\section{Sample and setting}

The sample for this study (number $[N]=212$ ) consisted of patients who completed the 5-year questionnaire booklet as part of the Heart Transplant Quality of Life Outcomes Study. ${ }^{26}$ The mean age of the sample was 54 years at the time of transplantation; $92 \%$ were Caucasian, and $80 \%$ were male. Patients were fairly well educated (mean: 14 years), and most patients had private health insurance or Medicare (Table 1). The study inclusion criteria were age $\geq 21$ years, able to read and write English, and being physically able to participate. The pool of

Table I Sample descriptives $(\mathrm{N}=2 \mid 2)$

\begin{tabular}{ll}
\hline Variable & M (SD) or $\mathbf{n}(\%)$ \\
\hline Age, years & $59.99(9.89)$ \\
Males, $\mathrm{n}$ & $169(80 \%)$ \\
White, $\mathrm{n}$ & $196(92 \%)$ \\
Education, years & $14.17(2.68)$ \\
Married, $\mathrm{n}$ & $163(77 \%)$ \\
Employed, $\mathrm{n}$ & $61(29 \%)$ \\
Depression score & $80.0(25.0)$ \\
No physical functioning disability, $\mathrm{n}$ & $96(45 \%)$ \\
\hline
\end{tabular}

Notes: Depression score is measured with the Cardiac Depression Scale. Higher scores indicate more depression; a cut-off score $>100$ indicates severe depression and $>90$ indicates mild to moderate depression. For physical functioning disability, the physical dimension of the Sickness Impact Profile was used. A score of zero indicates no functional disability.

Abbreviations: $\mathrm{N}$, total number; $M$, mean; $\mathrm{n}$, sample number; SD, standard deviation. 
transplant patients from which our patients were recruited is described elsewhere. ${ }^{26}$ Data were collected from four medical centers: the University of Alabama at Birmingham; Rush University Medical Center; the Cleveland Clinic; and the University of California, Los Angeles.

\section{Instruments}

Four self-report instruments were used for this study because they best answered the study aim. The Heart Transplant Stressor Scale measures the perceived stressfulness of the transplant experience (81 questions). ${ }^{26}$ Patients check "yes" or "no" if they have a stressor. If they check "yes", patients rate the stressfulness of the stressor on a Likert scale of 0-3 from not stressful to very stressful. The stressors are categorized into six subscales: physical; psychosocial; self-care; family; work/school/financial; and hospital/ clinic. A higher score indicates more stress. Cronbach's alpha coefficients for the total scale (0.95) and for the six subscales (0.78-0.90) support internal consistency reliability.

The Quality of Life Index - Cardiac Version IV developed by Ferrans and Powers ${ }^{24}$ was used to measure patient satisfaction with different areas of life. Part 1 measures satisfaction $(1=$ very dissatisfied to $6=$ very satisfied $)$, and part 2 measures importance ( $1=$ very unimportant to $6=$ important). This 35-item instrument has four subscales: health/functioning; socioeconomic; psychologic; and significant others. Internal consistency reliability for the total scale was supported with Cronbach alpha's ranging from $0.73-0.99$ in 48 studies. $^{24}$ In the current study, the total score was used, with higher scores reflecting higher HRQOL. The Quality of Life Index - Cardiac Version IV served as the dependent variable in the subsequent mediation analyses.

The Social Support Index measures the structural aspects of the social support network, functional types of assistance received (emotional, tangible, and overall), and satisfaction ( $1=$ very satisfied to $4=$ very dissatisfied $)$ with the support. ${ }^{27}$ The tool has two parts. Part A assesses the number and composition of the interpersonal ties and satisfaction with the amount of help the patient receives with each of 15 illness-related tasks such as personal care, taking medications, and travel to the doctor. Part B assesses the frequency of attendance at social activities and we did not use this part for the current study. Scoring of part A of this instrument includes calculating the number of support providers and a mean level of satisfaction with the support for each of the 15 tasks. Scores are categorized into satisfaction with tangible and emotional support. A lower satisfaction score indicates more perceived satisfaction with support. Internal consistency reliability was supported: Cronbach's alpha for the overall tool was 0.84 and the subscales carried an alpha of 0.78 for tangible support and 0.69 for emotional support. ${ }^{26}$

The Jalowiec Coping Scale is a widely used instrument that examines the use and perceived effectiveness of 60 coping strategies. ${ }^{28}$ Patients are asked to rate their coping use and effectiveness based on the stresses of the illness at the current time. Patients rate the use of coping strategies on a four-point Likert rating scale (ranging from $0=$ never used to $3=$ often used) and the perceived effectiveness of the strategies (ranging from $0=$ not helpful to $3=$ very helpful). This instrument has eight subscales: confrontive; evasive; optimistic; fatalistic; emotive; palliative; supportant; and self-reliant. These subscales are further categorized into positive and negative coping styles, with higher scores reflecting greater use of either positive and negative coping. Both mean use and effectiveness scores can be derived for each of the eight subscales. Reliability and validity is widely supported in the literature. ${ }^{28}$ For this study, positive coping effectiveness was used as one of the mediator variables given the proactive nature of this variable and the potential underlying implications for interventions.

\section{Analysis}

The SAS software version 13.1 (SAS Institute Inc., Cary, $\mathrm{NC}$, USA) was used for the descriptive and regression data analyses. A $P$-value $<0.05$ was considered statistically significant. To address the study aim, the following tests for mediation, according to Baron and Kenny, ${ }^{29}$ were performed. Briefly, a mediation analysis is used to test whether the influence of an independent variable on a dependent variable of interest is fully or partially explained by the mechanisms of a third variable (ie, the mediator). For example, the relationship between high levels of stress and cancer may be mediated by external risk factors such as smoking and poor diet, which are more prevalent among those with higher levels of stress. ${ }^{11,12}$ In the current study, to test for the mediation effect of satisfaction with social support on the association between perceived stress and HRQOL, the following steps were conducted: 1) regressing the mediator (satisfaction with social support) on the independent variable (perceived stress); 2) regressing the outcome variable (HRQOL) on the independent variable (perceived stress); and 3) regressing HRQOL on both the mediator (satisfaction with social support) and the independent variable (perceived stress). To test for the mediation effect of positive coping effectiveness, the following steps were conducted: 1) regressing the mediator (positive coping effectiveness) on the independent variable (perceived stress); 2) regressing the outcome variable (HRQOL) on the independent variable (perceived stress); and 
3) regressing HRQOL on both the mediator (positive coping effectiveness) and the independent variable (perceived stress). In order to control for the potential confounding effect of age and education level on the associations among variables, these two demographic factors were forced as covariates in all regression steps of each of the mediation analyses.

\section{Results}

\section{Satisfaction with social support}

A series of regression equations was used to test the mediating effect of satisfaction with social support on the relationship of stress and HRQOL at 5 years after heart transplantation. The first step revealed a significant relationship between satisfaction with social support and perceived stress ( $\beta=0.236 ; P<0.001$ ), indicating that less satisfaction with social support was associated with more perceived stress. The second step revealed a significant inverse relationship between perceived stress and HRQOL $(\beta=-0.578 ; P<0.001)$, indicating that subjects with better HRQOL experienced less perceived stress. Perceived stress explained $32 \%$ of the variance in HRQOL $\left(R^{2}\right.$ change $\left.=0.32 ; P<0.001\right)$. The final step in the mediation showed that together, satisfaction with social support and perceived stress (and its covariates) explained $46 \%$ of the variance in HRQOL $\left(R^{2}=0.464 ; P<0.001\right)$. The effect of perceived stress on HRQOL was smaller in step three ( $\beta=-0.489 ; P<0.001)$ than in step two $(\beta=-0.578 ; P<0.001)$; however, it remained significant, indicating that satisfaction with social support partially mediated the effects of perceived stress on HRQOL (Figure 1). In addition, subsequent analyses indicated that when examined separately, satisfaction with both emotional and tangible social support also partially mediated the relationship between perceived stress and HRQOL (Figures 2 and 3).

\section{Coping effectiveness}

To examine whether positive coping effectiveness mediated the relationship between perceived stress and HRQOL

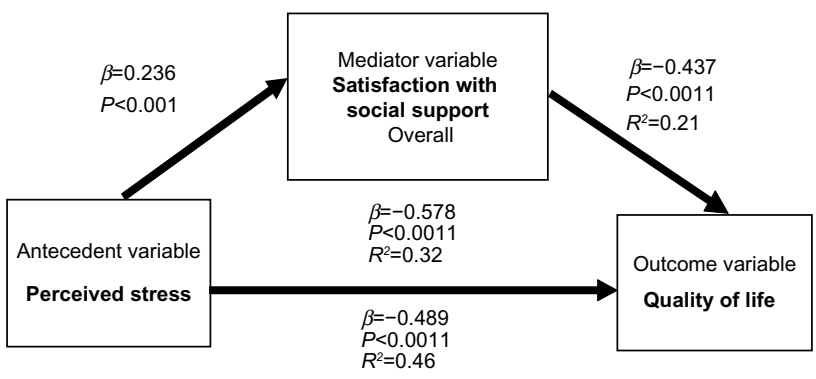

Figure I Mediation of overall satisfaction with social support on perceived stress and health-related quality of life (number $=212$ ).

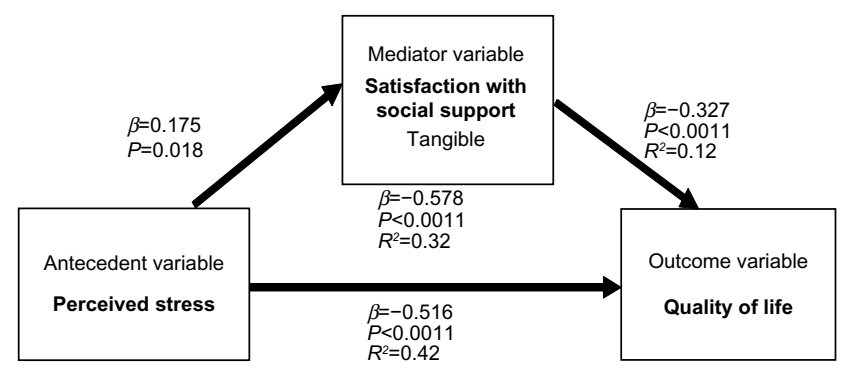

Figure 2 Mediation of satisfaction with tangible social support on perceived stress and health-related quality of life (number $=212$ ).

at 5 years after heart transplantation, a series of regression equations was used to test the mediating effect of coping. The first step revealed a significant inverse relationship between positive coping effectiveness and perceived stress $(\beta=-0.269$; $P<0.001$ ), indicating that those subjects who perceived more positive coping effectiveness had less perceived stress. The second step in the test for mediation revealed a significant inverse relationship between HRQOL and perceived stress; as indicated earlier, subjects with better HRQOL experienced less perceived stress. Perceived stress explained $32 \%$ of the variance in HRQOL $\left(R^{2}=0.32 ; P<0.001\right)$. The final step in the mediation showed that together, positive coping effectiveness and perceived stress (along with covariates) explained $36 \%$ of the variance in HRQOL $\left(R^{2}=0.355 ; P<0.001\right)$. The effect of perceived stress was smaller in step three $(\beta=-0.483$; $P<0.001)$ than in step two $(\beta=-0.578 ; P<0.001)$, yet it remained significant; therefore, it was concluded that positive coping effectiveness partially mediated the effects of perceived stress on HRQOL (Figure 4).

\section{Discussion}

Our study findings are a step toward further understanding the relationships among social support, perceived stress, and positive coping effectiveness, and their relationship to HRQOL over the long term after heart transplantation.

Both emotional and tangible social support partially mediated the relationship between stress and HRQOL, indicating

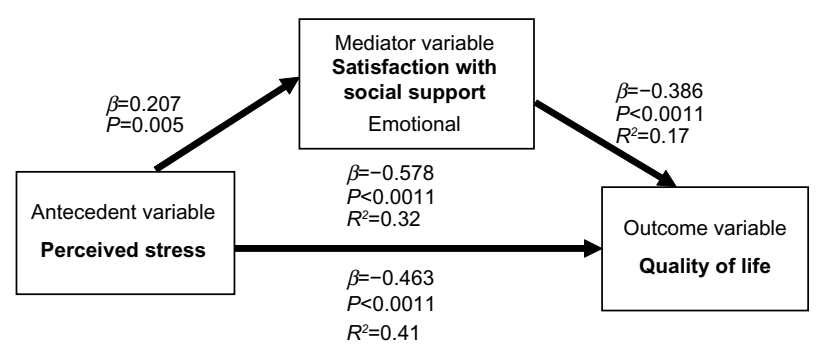

Figure 3 Mediation of satisfaction with emotional social support on perceived stress and health-related quality of life (number $=212$ ). 


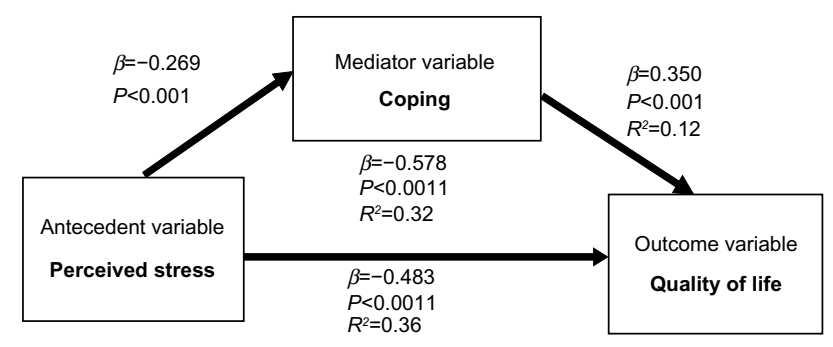

Figure 4 Mediation of coping effectiveness on perceived stress and health-related quality of life (number $=2 / 2$ ).

that social support plays an important role in the HRQOL of heart transplant patients. Patients had less stress if they were satisfied with their emotional and tangible support. At 5 years after transplant, the ability to confide in someone and one's closeness to others continues to be important. ${ }^{27}$ Even after the initial busy postoperative period when patients have increased contact with family and the transplant team, the need for closeness helps lessen stressful events that arise later after transplantation. The same is true for tangible support; having assistance with financial support or resources mediates stress over the long term after transplant. For example, $\mathrm{Wu}$ et $\mathrm{al}^{30}$ found that medication adherence mediated the relationship between marital status and survival in heart failure patients, implying that the social support provided by a spouse positively impacts medication adherence.

In addition, positive coping effectiveness partially mediated the association between perceived stress and HRQOL, demonstrating that effective positive coping strategies are necessary for impacting stress and HRQOL at 5 years posttransplant. This finding is similar to other study findings in patients with other chronic diseases. ${ }^{12,17}$ Furthermore, in a recent study, Grady et $\mathrm{al}^{31}$ reported that heart transplant patients who used negative coping strategies tended to have more stress, in terms of both frequency and intensity. This present study is an extension of those findings testing for a mediation relationship.

This study had limitations. Our study was limited by examining these data at only one time point (cross-sectional analyses of the 5-year post-transplant cohort). Thus, the interpretations of the mediation analyses could have been influenced by this, such that bidirectional associations between stress, coping, and social support may exist, which we were not able to examine in our study. For example, it may be instead that people with high levels of social support and coping skills may perceive events as being less stressful. Nonetheless, this study does provide useful implications for the associations between these variables. Another limitation of this study was that our sample was mostly white and had medical insurance; however, the sample was geographically diverse. This time frame had the largest sample size. Satisfaction with social support and coping may change across time, depending on life situations. We measured satisfaction with social support and coping in heart transplant recipients who survived to 5 years after transplant, which may introduce bias into our findings. Further, there may be unique psychosocial issues that may emerge immediately after heart transplantation (for example, 1-2 years post-transplantation), and this information may have been missed in the 5-year examination. With this said, the 5-year snapshot gives the clinician important information to assess the psychosocial status of their patients long-term following transplantation.

As health care professionals continue to care for patients long-term after transplantation, it is important to understand the subtle differences that may change over the years in regards to the relationships of satisfaction with social support, stress, effective coping, and HRQOL. By the time patients and families reach the 5-year time period, clinic visits and transplant team surveillance are less frequent compared to the time period immediately post-transplant. Our findings suggest that it would be beneficial to continue monitoring patients over the long term with phone calls or using other methods such as social media or mail to assess their social support, coping, and stress. This simple intervention may help to improve HRQOL over the long term after transplantation.

\section{Conclusion}

Our study provides evidence that psychosocial well-being continues to be important to patient outcomes over the long term after surgery. Satisfaction with social support continues to be an essential component to the transplant recipient's life. Also, coping effectiveness is important to being adept at handling stress over the long term after transplantation. Further investigation is needed to understand how health care providers can best deliver interventions aimed at dealing with social support issues and improving coping strategies, thereby leading to improved HRQOL and enhancements in clinical practice and patient outcomes.

\section{Acknowledgments}

This research was funded by the NIH (National Institute of Nursing Research, R01 \#NR005200); a grant-in-aid from the College of Nursing, Rush University; intramural funding from the Rush Heart Institute, Rush University Medical Center; and intramural funding from Northwestern University, Department of Surgery, Division of Cardiac Surgery. 


\section{Disclosure}

The authors report no conflicts of interest in this work.

\section{References}

1. George JF, Taylor DO, Blume ED, et al. Minimizing infection and rejection death: clues acquired from 19 years of multi-institutional cardiac transplantation data. J Heart Lung Transplant. 2011;30(2): 151-157.

2. Lund LH, Edwards LB, Kucheryavaya AY, et al; International Society for Heart and Lung Transplantation. The Registry of the International Society for Heart and Lung Transplantation: Thirtieth Official Adult Heart Transplant Report - 2013; focus theme: age. J Heart Lung Transplant. 2013;32(10):951-964.

3. Dew MA, Myaskovsky L, Switzer GE, DiMartini AF, Schulberg HC, Kormos RL. Profiles and predictors of the course of psychological distress across four years after heart transplantation. Psychol Med. 2005;35(8):1215-1227.

4. Huang CY, Hsu MC, Hsu SP, Cheng PC, Lin SF, Chuang CH. Mediating roles of social support on poststroke depression and quality of life in patients with ischemic stroke. J Clin Nurs. 2010;19(19-20):2752-2762.

5. DiMatteo MR. Social support and patient adherence to medical treatment: a meta-analysis. Health Psychol. 2004;23(2):207-218.

6. Vyavaharkar M, Moneyham L, Tavakoli A, et al. Social support, coping, and medication adherence among HIV-positive women with depression living in rural areas of the southeastern United States. AIDS Patient Care STDS. 2007;21(9):667-680.

7. Sayers SL, Riegel B, Pawlowski S, Coyne JC, Samaha FF. Social support and self-care of patients with heart failure. Ann Behav Med. 2008;35(1):70-79.

8. House J. Work Stress and Social Support. Reading, MA: Addison-Wesley; 1981.

9. Cohen S, Syme SL. Issues in the study and application of social support. In: Social Support and Health. San Francisco, CA: Academic Press; 1985:3-22.

10. Friedman MM, King KB. The relationship of emotional and tangible support to psychological well-being among older women with heart failure. Res Nurs Health. 1994;17(6):433-440.

11. Deno M, Tashiro M, Miyashita M, et al. The mediating effects of social support and self-efficacy on the relationship between social distress and emotional distress in head and neck cancer outpatients with facial disfigurement. Psychooncology. 2012;21(2):144-152.

12. Kim J, Han JY, Shaw B, McTavish F, Gustafson D. The roles of social support and coping strategies in predicting breast cancer patients' emotional well-being: testing mediation and moderation models. $J$ Health Psychol. 2010;15(4):543-552.

13. Closa León T, Nouwen A, Sheffield D, Jaumdally R, Lip GY. Anger rumination, social support, and cardiac symptoms in patients undergoing angiography. Br J Health Psychol. 2010;15(Pt 4):841-857.

14. Lazarus RS, Folkman S. Stress, Appraisal, and Coping. New York, NY: Springer Publishing Company; 1984.
15. Cobb S. Presidential Address-1976. Social support as a moderator of life stress. Psychosom Med. 1976;38(5):300-314.

16. McConnell D, Breitkreuz R, Savage A. From financial hardship to child difficulties: main and moderating effects of perceived social support. Child Care Health Dev. 2011;37(5):679-691.

17. Goetzinger AM, Blumenthal JA, O'Hayer CV, et al. Stress and coping in caregivers of patients awaiting solid organ transplantation. Clin Transplant. 2012;26(1):97-104.

18. Nilsson M, Forsberg A, Lennerling A, Persson LO. Coping in relation to perceived threat of the risk of graft rejection and Health-Related Quality of Life of organ transplant recipients. Scand J Caring Sci. 2013;27(4):935-944.

19. García-Alberca JM, Cruz B, Lara JP, et al. Disengagement coping partially mediates the relationship between caregiver burden and anxiety and depression in caregivers of people with Alzheimer's disease. Results from the MÁLAGA-AD study. J Affect Disord. 2012;136(3):848-856.

20. Dardas LA, Ahmad MM. Coping strategies as mediators and moderators between stress and quality of life among parents of children with autistic disorder. Stress Health. Epub July 19, 2013.

21. Holahan CJ, Moos RH, Holahan CK, Brennan PL. Social support, coping, and depressive symptoms in a late-middle-aged sample of patients reporting cardiac illness. Health Psychol. 1995;14(2):152-163.

22. House JS, Kahn RL, McLeod JD, Williams D. Measures and concepts of social support. In: Cohen S, Syme SL, editors. Social Support and Health. San Diego, CA: Academic Press; 1985:83-108.

23. Taylor MV, Bohachick P, Sereika SM, Schlenk EA, Brown C, Burke LE. Abstract 1731: Social support, personal control, and psychological functioning among individuals with heart failure. Circulation. 2007;116:II_365.

24. Ferrans CE, Powers MJ. Psychometric assessment of the Quality of Life Index. Res Nurs Health. 1992;15(1):29-38.

25. Spiker B, Revicki DA. Taxonomy of quality of life. In: Spiker B, editor. Quality of Life and Pharmacoeconomics in Clinical Trials. Philadelphia, PA: Lippincott-Raven Publishers; 1996:25-32.

26. Grady KL, Naftel DC, Kobashigawa J, et al. Patterns and predictors of quality of life at 5 to 10 years after heart transplantation. J Heart Lung Transplant. 2007;26(5):535-543.

27. White-Williams C, Grady KL, Myers S, et al. The relationships among satisfaction with social support, quality of life, and survival 5 to 10 years after heart transplantation. J Cardiovasc Nurs. 2013;28(5):407-416.

28. Jalowiec A. The Jalowiec Coping Scale. In: Strickland OL, DiIorio C, editors. Measurement of Nursing Outcomes: Self Care and Coping. New York, NY: Springer Publishing Company; 2003.

29. Baron RM, Kenny DA. The moderator-mediator variable distinction in social psychological research: conceptual, strategic, and statistical considerations. J Pers Soc Psychol. 1986;51(6):1173-1182.

30. Wu JR, Lennie TA, Chung ML, et al. Medication adherence mediates the relationship between marital status and cardiac event-free survival in patients with heart failure. Heart Lung. 2012;41(2):107-114.

31. Grady KL, Wang E, White-Williams C, et al. Factors associated with stress and coping at 5 and 10 years after heart transplantation. $J$ Heart Lung Transplant. 2013;32(4):437-446.
Nursing: Research and Reviews

\section{Publish your work in this journal}

Nursing: Research and Reviews is an international, peer-reviewed, open access journal publishing original research, reports, reviews and commentaries on all aspects of nursing and patient care. These include patient education and counselling, ethics, management and organizational issues, diagnostics and prescribing, economics and

resource management, health outcomes, and improving patient safety in all settings. The manuscript management system is completely online and includes a very quick and fair peer-review system. Visit http://www.dovepress.com/testimonials.php to read real quotes from published authors.

\section{Dovepress}

Submit your manuscript here: http://www.dovepress.com/nursing-research-and-reviews-journal 